\title{
L'Enseignement des langues par la tâche : le cas d'un cours visant les compétences informationnelles
}

\author{
Gabriel Michaud \\ Université McGill \\ Canada \\ Annie Desaulniers \\ Juliane Bertrand \\ Université du Québec à Montréal \\ Canada
}

\section{Introduction}

Le Québec reçoit environ 50000 immigrants chaque année et, de ce nombre, quelque 60-65\% ne maîtrisent pas suffisamment le français pour occuper un travail ou entreprendre des études universitaires. Ils suivent donc une formation linguistique pour favoriser leur insertion dans la société québécoise (Ferretti). Celle-ci peut prendre différentes formes, mais les principales options qui s'offrent à eux sont soit une série de cours de francisation offerts par le Ministère de l'Immigration, de la Diversité et de l'Inclusion (MIDI), soit l'inscription à un programme universitaire en langue seconde. Si les cours de langues secondes permettent aux apprenants d'acquérir de bonnes connaissances langagières et culturelles, certains d'entre eux se trouvent dépourvus au moment d'intégrer une formation universitaire de premier, deuxième ou troisième cycle ou une profession dans un nouveau contexte en raison, notamment, d'un manque de connaissances relativement aux compétences informationnelles requises pour entreprendre des études supérieures (p. ex. rédaction de rapports de recherche, présentation à caractère scientifique ou professionnel, travail d'équipe). Ces compétences informationnelles, définies par l'UNESCO et, au Québec, par le Plan de développement des compétences informationnelles (PDCI) constituent un ensemble de types de tâches que doit être capable d'accomplir une personne pour se documenter, produire des travaux et porter un jugement critique sur l'information et ce, tant dans les sphères scolaire, professionnelle et personnelle.

Devant ce constat, l'École de langues de l'Université du Québec à Montréal (UQAM) a créé le cours Français par projets qui vise à offrir aux étudiants du niveau 8 de l'Échelle québécoise des niveaux de compétence en français des personnes immigrantes adultes (Ministère de l'Immigration et des Communautés culturelles) ou B2 du Cadre européen commun de référence sur les langues (Conseil de l'Europe) une formation axée sur le développement de compétences scientifiques et professionnelles ainsi que de compétences langagières contextualisées (Bertrand et Desaulniers).

Dans l'article, il sera premièrement question des fondements théoriques sur lesquels s'appuie la conception du cours, à savoir l'enseignement des langues par la tâche. Nous présenterons ensuite la façon dont ont été incarnés les principes de l'enseignement par la tâche dans le cadre de la création du cours. Nous insisterons particulièrement sur la façon dont se déploie concrètement l'enseignement en salle de classe.

\section{L'enseignement des langues par la tâche}

Traditionnellement, l'enseignement des langues était vu comme la transmission décontextualisée de savoirs grammaticaux et lexicaux que les apprenants d'une langue seconde devaient maîtriser les uns à la suite des autres et intégrer de façon cumulative (Long).

Avec l'avènement de l'approche communicative à partir de la fin des années 70 , la conception de la langue s'est transformée et a plutôt été considérée comme un outil permettant la communication. Ce changement de paradigme a également trouvé écho dans les salles de classe où des situations plus proches de la vie réelle des apprenants étaient intégrées dans les programmes d'enseignement, par exemple, aller chez le médecin, faire son épicerie, passer un entretien d'embauche.

Toutefois, malgré les innovations concernant le rôle de la langue, plusieurs auteurs ont remarqué que la méthodologie d'enseignement continuait à suivre une structure traditionnelle : présentations de notions, suivies 
Michaud, Gabriel; Desaulniers, Annie et Juliane Bertrand. «L'Enseignement des langues par la tâche : le cas d'un cours visant les compétences informationnelles.» Nouvelle Revue Synergies Canada, №11 (2018)

d'exercices de systématisation et d'un réemploi des notions étudiées dans un contexte communicatif (Bygate, 384; Bygate et Samuda 56; Long 19; Richer 160). Selon Nunan (Learner-Centered 131), l'enseignement des langues par la tâche permet d'incarner sur le plan méthodologique les visées philosophiques de l'approche communicative qui conçoit la langue comme un outil de communication.

Comme son nom l'indique, l'enseignement des langues par la tâche utilise la tâche comme unité d'enseignement. Il s'agit de proposer des tâches communicatives pertinentes et significatives à un groupe d'apprenants donné qui leur permettront d'apprendre une langue seconde dans un contexte reproduisant, à divers degrés, des situations de la vie réelle.

Plusieurs définitions différentes de tâche ont été proposées (p. ex. Nunan Designing Tasks 10; Skehan 95; Van den Branden 4) qui s'entendent sur le fait que, dans le cadre d'une tâche, l'accent doit être mis sur le sens et la forme doit être au service de la tâche. Devant cette multitude de définitions, Ellis (223) a énuméré quatre critères qui doivent être respectés : l'accent doit être mis sur le sens, les apprenants ont un but à accomplir, ils doivent principalement utiliser leurs propres ressources pour accomplir la tâche et les objectifs de la tâche ne doivent pas être linguistiques.

Par conséquent, une tâche ne doit pas être seulement un prétexte pour faire passer un point de grammaire : il faut qu'il y ait une motivation communicative et pertinente pour les apprenants.

Au sein du domaine de l'enseignement des langues par la tâche, deux visions s'affrontent : celle de Long et celle d'Ellis. Pour Long, l'enseignement de la forme, notamment la forme grammaticale et la syntaxe, doit absolument être réactif, c'est-à-dire que le besoin doit émerger de la tâche, notamment sous forme de rétroaction corrective. L'enseignant ne peut donc pas cibler au préalable des notions linguistiques.

Par contre, pour Ellis, il est possible de cerner certaines notions grammaticales qui sont susceptibles d'être nécessaires ou utiles à l'accomplissement d'une tâche, ce qu'il appelle une " tâche cible ». II insiste toutefois pour dire qu'il ne faut pas imposer l'utilisation obligatoire d'une notion spécifique, ce qui constituerait, selon lui, non pas une tâche, mais bien un exercice grammatical.

Comme il existe plusieurs définitions de «tâche », il existe également différentes architectures d'enseignement (pour plus de détails à ce sujet, voir Michaud). Norris (581-582), reconnaissant cette diversité, recense tout de même certains éléments communs à un programme d'enseignement par la tâche :

1) analyse des besoins;

2) sélection de tâches et séquence;

3) développement de matériel didactique;

4) enseignement;

5) évaluation;

6) évaluation de programme.

Dans les prochaines sections, nous expliquons la façon dont a été développé chacun de ces éléments pour la conception d'un cours.

\section{Analyse des besoins}

Pour assurer la pertinence d'un cours, Long (10) affirme que ce dernier doit s'appuyer sur une analyse des besoins des apprenants.

Dans le cas présent, ce sont des discussions informelles avec d'anciens diplômés du programme de français langue seconde qui ont motivé la création du cours. Ceux-ci ont fait part des obstacles auxquels ils faisaient face au moment d'intégrer un programme d'études universitaires ou un nouveau travail, notamment en ce qui 
concerne la réalisation de travaux scientifiques, la lecture de textes scientifiques en français, l'évaluation critique de la valeur d'un texte à l'oral ou à l'écrit et le fait de communiquer oralement sur un sujet spécialisé.

Ces résultats ont motivé la création d'un sondage qui a été mené auprès d'étudiants du programme de FLS pour mieux connaître la nature de leurs besoins sur le plan universitaire et professionnel. Parmi les résultats obtenus, à la question de savoir s'ils avaient déjà vécu l'expérience de mener une recherche, dans leur première langue ou dans une autre langue, sur les 43 étudiants ayant répondu au sondage, 20 (47\%) ont affirmé qu'ils l'avaient fait avant de commencer leurs études en français à l'UQAM et 23 (53\%) ont répondu que non. C'est donc un peu plus de la moitié de ces étudiants se destinant majoritairement à poursuivre des études universitaires au Québec qui n'ont jamais eu à utiliser de compétences informationnelles en vue de préparer un rapport de recherche.

Dans le même sondage, nous avons comparé les résultats d'une étude effectuée par le Ministère de l'Éducation, du Loisir et du Sport (MELS) portant sur les cinq compétences les plus recherchées par 1406 employeurs québécois et la valeur qu'accordent les étudiants à ces compétences. Dans cette étude, le terme compétence était employé d'une façon très large pouvant inclure toute dimension du travail. La figure 1 montre que, pour chacune de ces cinq compétences, les apprenants expriment dans une grande proportion le désir de les développer dans le cadre de leurs cours de français.

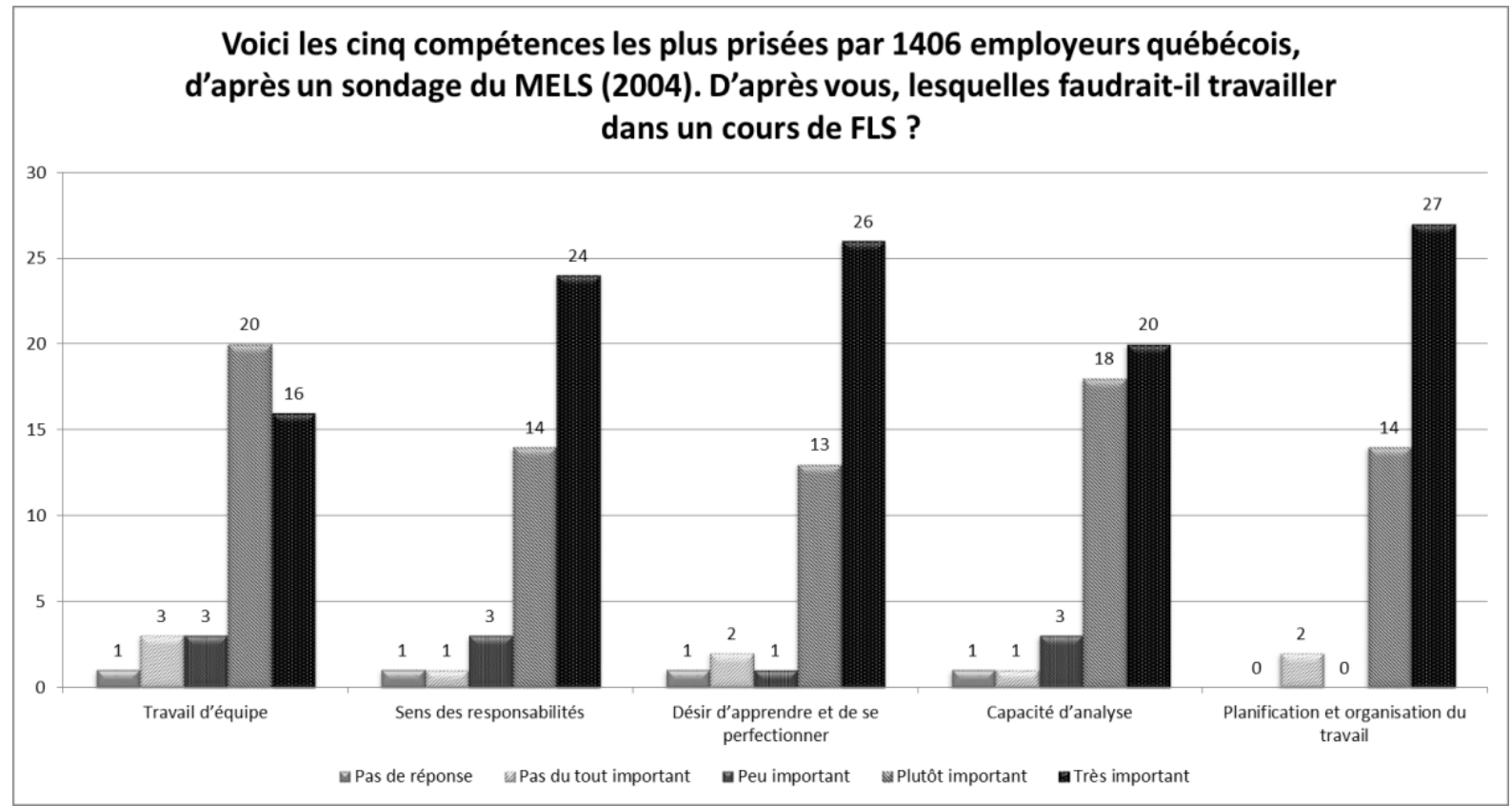

Fig. 1. Intérêt des étudiants à développer leurs compétences transversales en L2

En plus des discussions informelles et du sondage mené en classe, nous avons procédé à une analyse des principes édictés par des organismes gouvernementaux et des institutions internationales en ce qui concerne les compétences informationnelles à acquérir. Par exemple, l'UNESCO soutient que des connaissances liées à la maîtrise de l'information sont nécessaires pour que les personnes et les sociétés puissent posséder les savoirs essentiels à l'amélioration de leur vie quotidienne (Horton Jr). Parallèlement, au Québec, est mis sur pied le Plan de développement des compétences informationnelles (PDCl) qui propose sept normes énonçant les compétences informationnelles attendues chez des étudiants universitaires (Université du Québec). Les normes touchent à la reconnaissance du besoin d'information, à l'accès efficace aux ressources disponibles, à l'évaluation critique des sources, au développement de nouvelles connaissances, à la réalisation de travaux, à la compréhension des enjeux culturels et éthiques liés au réemploi de l'information et à la mise à jour continuelle des connaissances. Ces normes doivent donc être intégrées à un cours visant le développement de compétences informationnelles. 
Michaud, Gabriel; Desaulniers, Annie et Juliane Bertrand. « L'Enseignement des langues par la tâche : le cas d'un cours visant les compétences informationnelles.» Nouvelle Revue Synergies Canada, №11 (2018)

En somme, l'analyse des besoins a permis de mettre en relation les besoins subjectifs exprimés par les étudiants dans le cadre d'un sondage et de discussions informelles ainsi que des besoins objectifs mis de l'avant par des organismes gouvernementaux. II en est ressorti l'importance de développer des compétences informationnelles permettant de mener à bien des études universitaires par des apprenants du français langue seconde en contexte québécois.

\section{Sélection de tâches et séquence}

Cette première phase a permis d'orienter le développement des contenus visés par le cours. Une tâche générale a été retenue qui permet de répondre à une série de besoins cernés dans l'analyse des besoins : la réalisation d'un rapport de recherche sur une question scientifique. Cette tâche se compose d'une série de sous-tâches : effectuer une recherche documentaire, lire et évaluer des articles scientifiques, produire un rapport de recherche écrit, faire une communication scientifique ou professionnelle orale lors d'un colloque. Pour cibler les compétences à acquérir dans le cadre de ces tâches, nous nous sommes fiés aux indicateurs énoncés dans le PDCl.

II a tout d'abord fallu procéder à la transposition didactique de ces indicateurs de façon à concevoir des activités pédagogiques adaptées à l'âge de l'apprenant, au niveau de scolarité, aux ressources disponibles et au niveau de compétence langagière en langue seconde (compréhension et production orales et écrites).

Le tableau 1 illustre le processus de didactisation des indicateurs du PDCl.

\begin{tabular}{|l|l|l|}
\hline Norme ou indicateur & Compétence en FLS & Activités à envisager \\
\hline $\begin{array}{l}\text { 1.1 Définir et exprimer clairement son } \\
\text { besoin d'information }\end{array}$ & $\begin{array}{l}\text { Formuler grammaticalement une } \\
\text { question cohérente }\end{array}$ & $\begin{array}{l}\text { Leçon et activités sur la phrase de } \\
\text { type interrogatif }\end{array}$ \\
\hline $\begin{array}{l}\text { 2.3 Repérer l'information en utilisant } \\
\text { recherche }\end{array}$ & $\begin{array}{l}\text { Manipuler des synonymes pour } \\
\text { chercher des mots-clés adaptés à } \\
\text { divers sites de recherche }\end{array}$ & $\begin{array}{l}\text { Présentation du dictionnaire } \\
\text { unilingue, travail sur les familles de } \\
\text { mots }\end{array}$ \\
\hline $\begin{array}{l}5.5 \text { Communiquer efficacement aux } \\
\text { autres le fruit de son travail }\end{array}$ & Présenter un résumé oral & $\begin{array}{l}\text { Discussion informelle, participation à } \\
\text { un mini-colloque }\end{array}$ \\
\hline
\end{tabular}

Tableau 1. Transposition des indicateurs en activités pour la classe de FLS

Par la suite, le déroulement complet du cours a été prévu. Le cours de Français par projets offert à l'UQAM, d'une durée de 15 semaines, se divise en quatre phases. D'abord, la première phase, d'ordre documentaire, comprend la formation d'équipes de quatre étudiants partageant un même intérêt professionnel, la formulation d'une question de recherche ainsi que des ateliers sur les compétences informationnelles québécoises élaborés en collaboration avec une bibliothécaire pour la recherche d'articles et l'évaluation critique des sources. Pour accomplir ces tâches, l'étudiant s'appuie sur des notions telles que la phrase interrogative et la synonymie.

La deuxième phase consiste en une initiation à la lecture d'articles universitaires en L2 et en l'élaboration d'un plan détaillé. Dans la troisième phase, des techniques pour la rédaction, comme la reformulation à l'aide de synonymes et de mots de même famille ou le choix de structures de phrase pour citer la source et introduire le discours rapporté, sont présentées. Au cours de cette phase, l'étudiant rédige individuellement un premier jet d'une section du travail collectif et présente informellement à l'oral le travail de son équipe pour récolter des commentaires.

La dernière phase est la phase de communication plus formelle de l'information acquise, où les étudiants devront procéder à la finalisation du travail écrit en équipe et à une présentation orale lors d'un colloque estudiantin ouvert au public, réinvestissant ici toutes leurs ressources langagières. Le contenu de ces blocs est perméable, donc il y a des retours sur les apprentissages et des activités anticipant ce qui sera approfondi plus tard.

\section{Développement de matériel didactique}

Comme il s'agit d'un cours par la tâche qui vise les compétences informationnelles, notamment la capacité de trouver et d'évaluer des ressources documentaires, la majorité du matériel utilisé, principalement des articles 
Michaud, Gabriel; Desaulniers, Annie et Juliane Bertrand. « L'Enseignement des langues par la tâche : le cas d'un cours visant les compétences informationnelles.» Nouvelle Revue Synergies Canada, №11 (2018)

scientifiques, sera trouvé par les étudiants. Puisque chaque équipe travaille sur une question en lien avec ses intérêts professionnels, le matériel utilisé est très varié au sein d'une même classe.

Par contre, afin de bien préparer et d'accompagner les étudiants, plusieurs fiches d'information ont été conçues conjointement par les enseignantes et la bibliothécaire responsable du secteur des langues secondes de I'UQAM ${ }^{1}$. Deux critères ont servi de point de repère pour cibler les compétences devant faire l'objet d'un enseignement: la pertinence culturelle et la complexité des interférences linguistiques. Par la suite, la bibliothécaire a élaboré une série de matériel se déclinant en cinq volets spécifiquement adaptés à des étudiants avancés en FLS, soit la délimitation du sujet, l'élaboration du plan de concepts, la recherche de documents à l'aide de Repère, Google Scholar et Virtuose - le premier étant une base de données regroupant les revues spécialisées francophones, le troisième étant le catalogue des bibliothèques de l'UQAM - ainsi que l'évaluation des sources et la fiche de lecture. Un dernier volet, celui de l'intégrité académique, est traité à l'aide de stratégies pour éviter le plagiat.

Si ces activités sont développées en collaboration entre les enseignantes de FLS et la bibliothécaire, c'est à cause de la volonté de créer un cours où le travail sur les compétences informationnelles est intimement lié au travail sur les compétences langagières. De plus, le fait que ce matériel soit abrité sur le site Internet des bibliothèques de l'UQAM contribue à faire comprendre à l'étudiant que les tâches liées à la recherche sont une part inhérente de la vie universitaire et non une réalité spécifique à leurs cours de langue.

Du point de vue linguistique, les étudiants auront à formuler des questions de recherche, donc des exercices sur la forme interrogative ont été prévus. De plus, des modèles de paragraphe et de rapports de recherche ont été créés. Grâce à ces documents, les étudiants auront accès à des modèles pour réaliser les tâches.

En ce sens, l'approche retenue relativement à l'enseignement des compétences langagières comprend à la fois un enseignement proactif (c'est-à-dire que des explications et des exercices sur la forme interrogative ont été prévus) et un enseignement réactif donné sous forme de rétroaction corrective et de capsules grammaticales intégrées en fonction des besoins linguistiques manifestés par les apprenants au cours de l'accomplissement de la tâche (c'est-à-dire que l'enseignant fait un temps d'arrêt pour donner des explications de nature linguistique).

\section{Démarche pour l'enseignement des compétences informationnelles en FLS}

À présent, il sera question en détail du déploiement de l'enseignement en salle de classe, notamment du déroulement des tâches et des activités.

Dans la première partie de la session, la bibliothécaire est très présente et fournit un enseignement aux étudiants sur la façon de mener à bien une recherche documentaire. Après que les enseignantes du cours ont aidé les étudiants à construire leur question de recherche, elle présente le plan de concepts et explique son objectif, qui est de constituer une banque de mots pour trouver des textes appropriés sur Google Scholar ou dans d'autres bases de données et détaille la démarche pour remplir ce document.

Par exemple, à partir de la question de recherche Comment faciliter l'intégration des enfants immigrants dans le système scolaire québécois?, la bibliothécaire explique qu'il faut repérer les noms (intégration, enfant, système scolaire) et le nom dérivé de certains adjectifs (immigrant, Québec) et construire un tableau dans lequel chacun de ces noms sert d'en-tête à une colonne. Elle précise ensuite que, pour remplir le plan de concepts, il faut trouver des synonymes pour chacun de ces mots et les inscrire dans la colonne, sous le nom extrait de la question.

De leur côté, les enseignantes de FLS profitent de la préparation du plan de concepts pour initier les apprenants à la consultation de dictionnaires unilingues en ligne en leur faisant connaître le symbolisme identifiant les synonymes. Il s'agit d'une bonne occasion d'expliquer l'importance de vérifier si le mot est synonyme en contexte.

À partir de la question précédente, le mot intégration permet d'entamer ce travail. En cherchant ce mot dans le Petit Robert, l'étudiant constate que la définition est divisée en catégories d'emploi - mathématique, philosophique, économique, courant - dont seul le dernier peut correspondre au contexte de la question. Comme les synonymes possibles sont répartis selon les mêmes catégories, l'étudiant comprend qu'il ne peut pas utiliser quadrature ou filière comme synonyme dans le contexte de sa question. Pour le sens le plus approprié, le Petit 
Michaud, Gabriel; Desaulniers, Annie et Juliane Bertrand. « L'Enseignement des langues par la tâche : le cas d'un cours visant les compétences informationnelles.» Nouvelle Revue Synergies Canada, №11 (2018)

Robert propose trois synonymes, assimilation, fusion et insertion. Avec des étudiants en perfectionnement en FLS, il est pertinent d'aller plus à fond dans la démarche. Ils sont donc invités à cliquer sur assimilation, ce qui les renvoie à assimiler, qui est associé à l'idée de « traiter comme semblable, [...] confondre " (Petit Robert). Une brève discussion sur les valeurs des immigrants et des Québécois amène à la conclusion que, lorsqu'il est question d'intégration au Québec, ce n'est pas dans une visée assimilatrice, donc le mot est écarté de la liste des synonymes à maintenir dans le contexte spécifique de la question de recherche choisie. Ce choix peut être validé par une présentation de USITO, dictionnaire conçu au Québec, où l'on constate qu'assimilation n'est pas proposé comme synonyme d'intégration. Un tel type de travail sur le dictionnaire pourra être approfondi dans la phase de lecture de textes scientifiques à l'aide de cartes d'études de mots, un travail qui amène l'étudiant à analyser des mots issus des textes lus à l'aide du dictionnaire unilingue (Baron et Bertrand).

Grâce au travail combiné de la bibliothécaire et des enseignantes de FLS ainsi qu'à l'utilisation des outils technologiques mentionnés ci-haut, les étudiants peuvent donc développer leurs compétences en FLS dans un domaine de leur choix.

Après avoir reçu ces explications, l'équipe prépare son plan à partir de sa propre question de recherche, et cette recherche de synonymes lui permet d'étendre l'ampleur du vocabulaire lié au domaine professionnel. Puis, sont présentées les techniques pour trouver des documents scientifiques ou professionnels à partir d'un plan de concepts. Les étudiants reçoivent ensuite un enseignement sur les six critères pour évaluer la valeur scientifique de leurs sources. Il s'agit de la pertinence du document pour répondre à la question, de la fiabilité de l'édition, de la réputation de l'auteur, de l'objectivité de l'information, de l'exactitude des données et de l'actualité du document. Ce travail amène une réflexion sur les valeurs du milieu universitaire québécois.

Plus tard dans la session, dans la troisième phase, les principaux types de plagiat et les stratégies pour l'éviter sont présentés aux étudiants. Les enseignants mettent notamment l'accent sur la façon de citer indirectement un auteur. En effet, les étudiants d'une L2, compte tenu de leur connaissance limitée du vocabulaire et des structures syntaxiques de la L2, ont de la difficulté à présenter les idées d'autres auteurs dans leurs propres mots. Pour les aider à développer leur compétence en matière de reformulation, une activité en quatre temps est proposée. La première étape consiste à lire un paragraphe d'un texte informatif et à y surligner les mots les plus importants. Les étudiants réalisent que ce sont généralement les noms et les verbes, et quelquefois les adjectifs, qui importent le plus au sens du texte. Dans la deuxième étape, les étudiants prennent une feuille blanche sur laquelle ils doivent inscrire un synonyme ou un mot de la même famille de chacun des mots qu'ils ont surlignés. II s'agit d'une occasion de faire un retour sur l'emploi du dictionnaire unilingue. Quand cette étape est complétée, les étudiants doivent cacher le texte d'origine et essayer, de mémoire, de réécrire la même information à partir des mots qu'ils ont notés sur leur feuille blanche. Enfin, la quatrième étape consiste à ressortir le texte original, puis à lire les deux textes côte à côte en s'assurant que le contenu est le même, mais que la structure des phrases ne se ressemble pas trop. Grâce à ces quatre étapes, les étudiants arrivent à produire un texte bien reformulé.

Le colloque a lieu à la quatrième phase et conclut le cours. II respecte le format des principaux colloques scientifiques nord-américains, c'est-à-dire que chaque équipe dispose de vingt minutes pour présenter son sujet et chaque présentation est suivie d'une période de questions de dix minutes. Les séances sont organisées thématiquement, et ce sont les étudiants qui remplissent eux-mêmes l'ensemble des rôles nécessaires à la tenue d'un colloque : arbitrage des séances, organisation du comité d'accueil, des collations pour les pauses et le dîner, etc. II s'agit d'une activité authentique qui favorise une communication véritable et qui est annoncée sur Internet et dans les journaux et ouverte au public.

Trois facteurs favorisent grandement le succès de l'activité. Pour augmenter la confiance des étudiants, une présentation orale informelle dans les semaines précédentes au cours de laquelle ils reçoivent une rétroaction joue un rôle majeur. De plus, la correction préalable du fichier PowerPoint ou Prezi par une des enseignantes permet aux étudiants de valider leur contenu et d'être rassurés sur l'absence d'erreurs liées à la langue, du moins dans la présentation visuelle. Enfin, pour favoriser les échanges dans les dix minutes suivant chaque présentation, en écoutant les communications, les étudiants remplissent une grille qui est orientée vers la formulation de questions. La motivation des étudiants est suffisante pour qu'il n'y ait aucun temps mort pendant les périodes de questions.

Le fait de confier aux étudiants la préparation des pauses est une occasion de leur permettre de faire connaître un plat de leur pays d'origine. Ainsi, les étudiants sentent que leurs efforts sont récompensés par le plaisir du partage et de la découverte. 


\section{5. Évaluation}

À l'intérieur du cours, toutes les évaluations reposent sur les tâches réalisées par les étudiants. Le premier volet, celui du développement des compétences informationnelles, est évalué par le biais d'un portfolio à l'intérieur duquel l'étudiant regroupe tous les documents sur lesquels il a travaillé pendant la session : fiches remplies pour définir le sujet, plans de concepts établis pour la recherche bibliographique dans les bases de données, fiches d'évaluation de la pertinence des sources retenues, notes de lecture, brouillons, etc. La notation de ce portfolio repose sur la progression reflétée par les documents.

Le deuxième volet, celui de la présentation orale d'un sujet de recherche, est évalué à deux niveaux. D'abord, deux ou trois semaines avant le colloque, l'étudiant présente informellement l'état d'avancement du travail de son équipe à deux collègues de classe pour recevoir des suggestions. À ce stade, il est évalué uniquement sur son investissement dans l'échange. L'évaluation la plus importante est celle de la performance lors du colloque. Elle repose sur la capacité de l'étudiant de présenter sa partie de la communication de façon claire, tout en respectant la structure globale de l'exposé choisie par son équipe. La capacité d'utiliser un vocabulaire précis pour parler du sujet choisi tout en étant compréhensible par l'auditoire est aussi évaluée.

De son côté, le troisième volet, qui touche la préparation du rapport de recherche écrit, est évalué en deux temps. Premièrement, l'étudiant remet deux paragraphes rédigés en classe, qui sont évalués en fonction de leur structure et de la façon dont les sources y sont citées. Deuxièmement, l'équipe d'étudiants remet le travail final, qui est évalué selon la qualité du contenu et la précision langagière.

\section{6. Évaluation de programme : appréciation des étudiants}

Pour mieux connaître l'opinion des étudiants sur le cours décrit ci-dessus, un sondage a été réalisé en décembre 2016, un an après la mise en place du cours. Sur les 43 répondants, 36 étaient sur le point de terminer le cours et 7 l'avaient suivi durant l'une des sessions précédentes.

D'abord, les participants devaient indiquer leur niveau d'accord avec l'affirmation « Un cours de langue traditionnel est préférable à un cours axé sur la réalisation d'un projet » (fig. 2). Les positions sont très partagées : $47,4 \%$ expriment une préférence pour une approche plus traditionnelle et 52,6\% privilégient une approche axée sur des tâches.

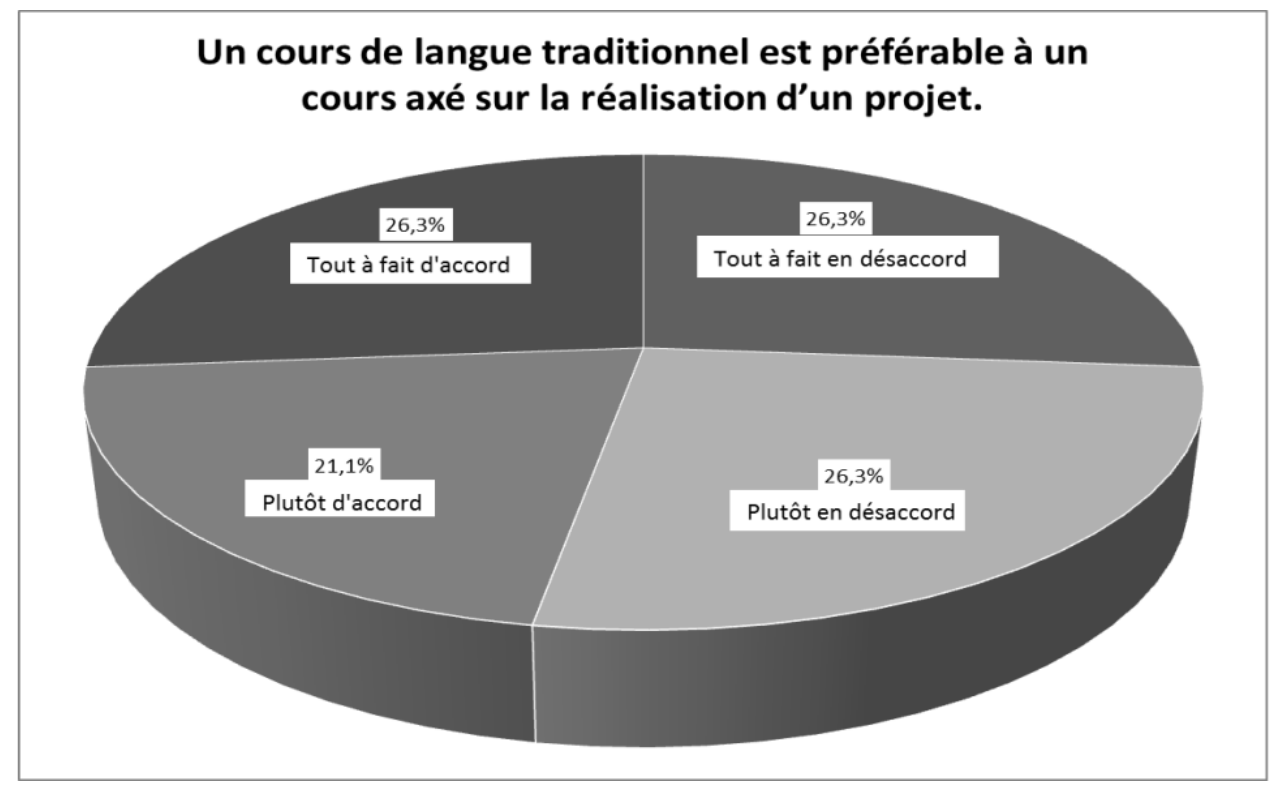

Fig. 2. Opinion des étudiants sur l'approche choisie

Par contre, pour la grande majorité des répondants, le fait de produire un projet en français a permis de développer leurs compétences langagières (fig. 3). Ainsi, plus de $80 \%$ sont plutôt ou tout à fait d'accord pour 
Michaud, Gabriel; Desaulniers, Annie et Juliane Bertrand. «L'Enseignement des langues par la tâche : le cas d'un cours visant les compétences informationnelles.» Nouvelle Revue Synergies Canada, №11 (2018)

dire que produire un projet en français leur a permis d'améliorer leur vocabulaire, leurs connaissances grammaticales, leurs aptitudes en lecture et leur confiance pour communiquer en français. II semble donc qu'à travers la réalisation des tâches, les étudiants aient développé l'ensemble de leurs compétences en FLS.

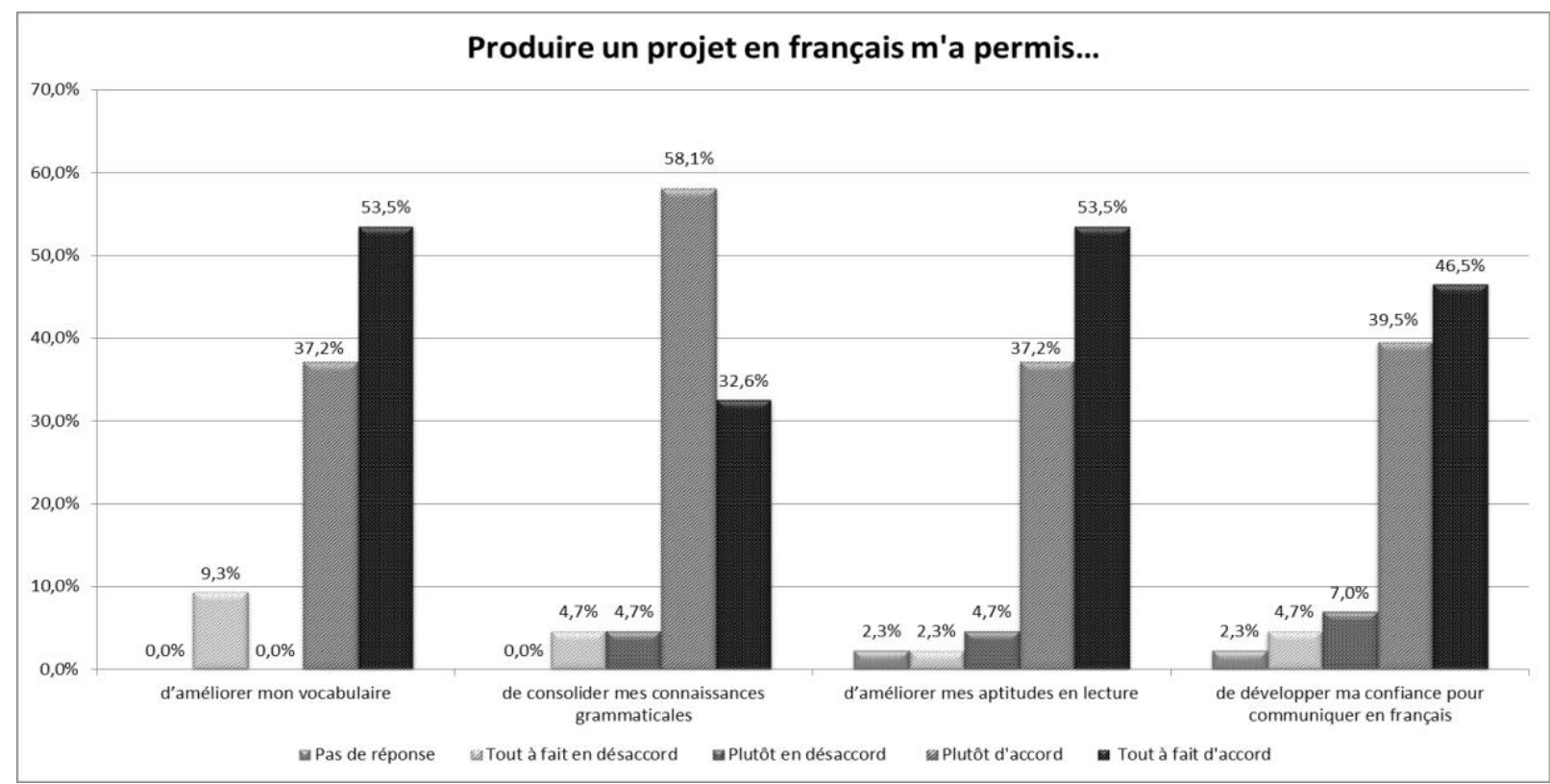

Fig.3. Perception des répondants sur leur progression

Concernant le cheminement à moyen ou long terme, la figure 4 indique que les participants ont la perception que le fait d'avoir produit un projet en français les aidera à atteindre leurs objectifs scolaires ou professionnels. Près de $70 \%$ des répondants sont plutôt ou tout à fait d'accord avec cette idée.

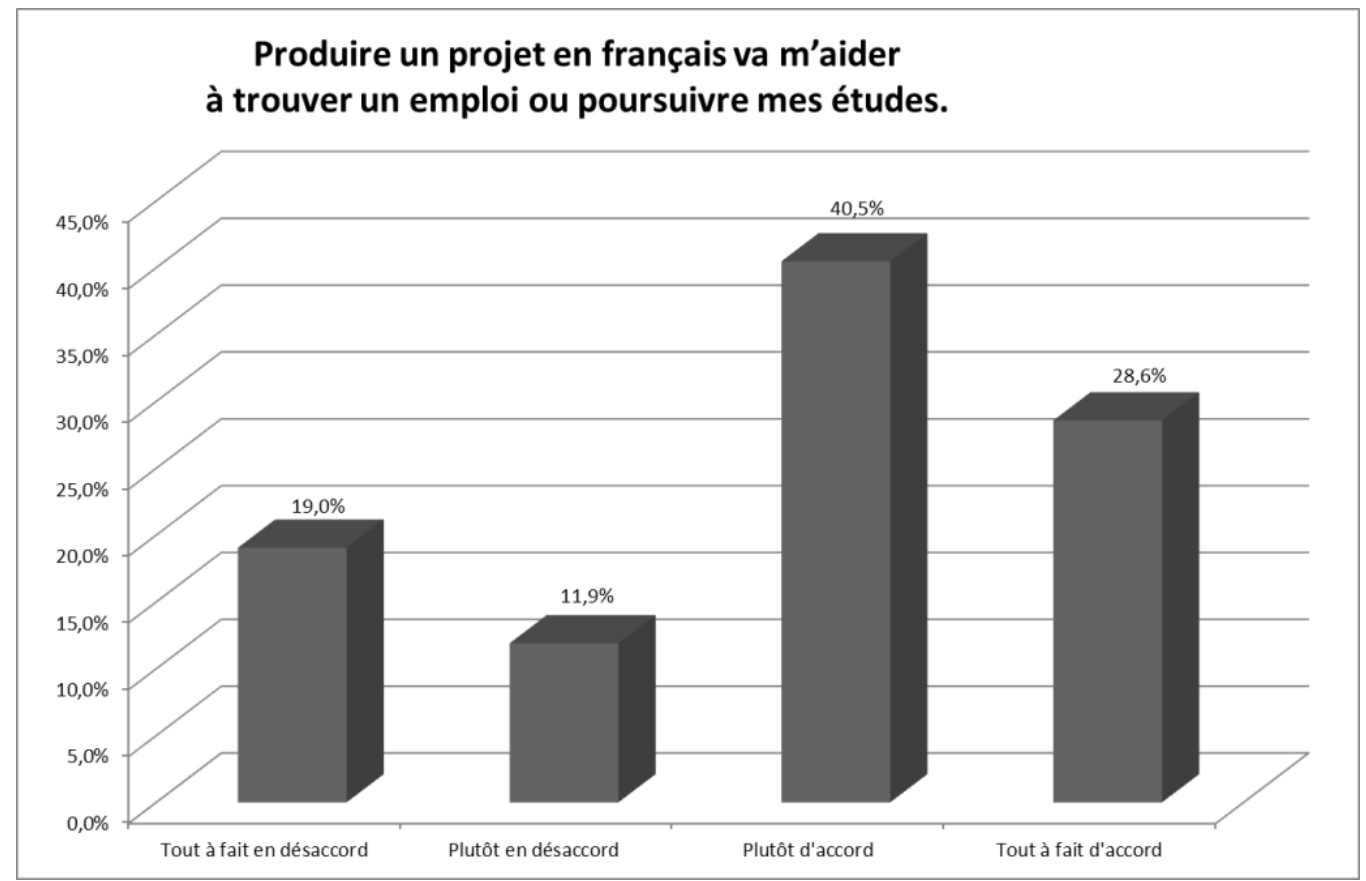

Fig. 4. Impact de la réalisation d'un projet en français sur la réalisation des objectifs 
Quant au fait de travailler en équipe pour la rédaction d'un rapport et la préparation d'un exposé oral (figure 5), $65 \%$ des participants affirment que c'est une expérience totalement positive. Parmi les avantages du travail en équipe, ils ont signalé l'amélioration de leurs stratégies de communication, la possibilité de partager des idées et de les enrichir au cours de discussions avec des collègues, l'aide mutuelle et l'apprentissage réciproque, le perfectionnement du français, l'amélioration de leurs capacités de planification, la possibilité de vaincre leurs peurs, l'occasion de vivre une première expérience de travail d'équipe, le développement de leurs qualités personnelles (vertu, patience, responsabilité), le respect des autres et la possibilité de se faire des amis.

Ceux qui ont jugé l'expérience de travail en équipe plutôt ou complètement négative (13\%) ont mentionné l'impression de perdre du temps pour communiquer, la difficulté de convaincre les autres de leurs idées, l'obligation de faire face à des malentendus, l'impression de moins en apprendre sur la langue, la crainte de recevoir une note plus faible, le sentiment que les consignes ne sont pas comprises par tous leurs coéquipiers ou la sensation que certains participants étaient irresponsables.

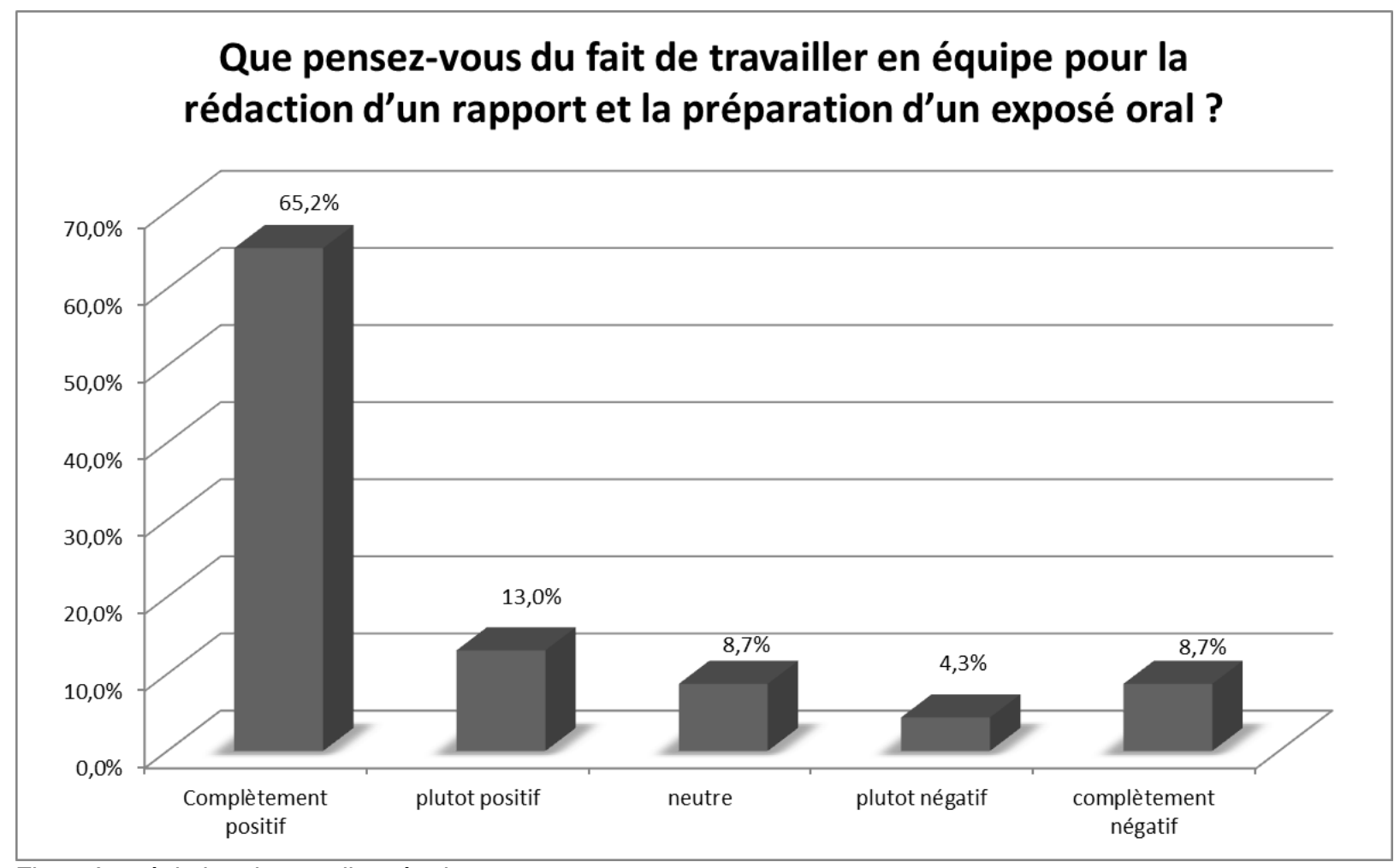

Fig. 5 Appréciation du travail en équipe

Ces données montrent que, pour la majorité des étudiants ayant répondu au sondage, la réalisation des tâches permettant de compléter leur projet est perçue positivement.

\section{En guise de conclusion : la progression des étudiants}

En somme, en s'appuyant sur les fondements de l'enseignement des langues par la tâche, le cours de Français par projets présente de grands avantages pour les étudiants de FLS adultes et scolarisés.

Les compétences développées touchent tous les aspects de la langue. Sur le plan de la compréhension écrite et du lexique, les étudiants commencent à assimiler le vocabulaire du domaine professionnel de leur choix. Sur le plan de l'interaction orale, ils mettent en pratique des stratégies pour communiquer vers la réalisation d'un but commun. Sur le plan de l'expression orale, l'expérience de présentation informelle pour l'échange de commentaires ainsi que de communication formelle dans le cadre d'un colloque estudiantin contribue à produire des locuteurs aptes à s'exprimer dans une variété de contextes. Enfin, sur le plan de l'expression écrite, la 
Michaud, Gabriel; Desaulniers, Annie et Juliane Bertrand. «L'Enseignement des langues par la tâche : le cas d'un cours visant les compétences informationnelles.» Nouvelle Revue Synergies Canada, №11 (2018)

rédaction d'un article de type scientifique formel et de documents de travail provisoires semi-formels permet à l'étudiant d'apprendre à adapter ses écrits à la situation de communication.

À tout cela s'ajoute le développement de la compétence à communiquer au sein d'une équipe interculturelle et celui des compétences informationnelles, deux autres éléments essentiels pour entreprendre des études universitaires ou travailler au Québec. II s'agit d'une expérience positive qui permet d'harmoniser les besoins langagiers des apprenants aux compétences universitaires et professionnelles dont ils auront besoin. L'enseignement des langues par la tâche, malgré certaines réticences soulevées par quelques étudiants, gagne donc à être développé dans le cadre d'un cours de niveau universitaire.

\section{Note}

${ }^{1}$ Les auteurs tiennent à remercier $\mathrm{M}^{\mathrm{me}}$ Pierrette Richèr pour son soutien précieux.

\section{Bibliographie}

Baron, Agnès et Juliane Bertrand. "Utiliser le dictionnaire monolingue en classe de langue. » Correspondance, vol. 18, nº 1, 2012 pp. 31-35.

Bygate, Martin. "Sources, developments and directions of task-based language teaching." The language learning journal, vol. 44, no 4, 2016, pp. 381-400.

Bertrand, Juliane et Annie Desaulniers. «Favoriser le développement des compétences informationnelles en langue seconde à travers la réalisation d'un projet personnalisé : création d'un cours universitaire en FLS. " Reflets, numéro spécial pour les 35 ans de l'AQEFLS - Le Français langue seconde en fête : mythes, réalités et partage de bonnes pratiques, 2016, pp. 273-281.

Conseil de l'Europe. Cadre européen commun de référence pour les langues - Apprendre, enseigner, évaluer, Didier, 2001.

Ellis, Rod. "Task-based language teaching: Sorting out the misunderstandings." International Journal of Applied Linguistics, vol. 19, no 3, 2009, pp. 221-246.

Ferretti, Jean. Le Québec rate sa cible. Les efforts du Québec en matière de francisation et d'intégration des immigrants : un portrait, Rapport de recherche de l'IRÉC, Montréal, Institut de recherche en économie contemporaine, 2016.

Horton Jr, Forest Woody. Introduction à la maîtrise de l'information, UNESCO, 2008, http://unesdoc.unesco.org/images/0015/001570/157020f.pdf.

Long, Mike. Second language acquisition and task-based language teaching. John Wiley \& Sons, 2014.

Michaud, Gabriel. « L'enseignement par la tâche. » Reflets du 35e Congrès de l'AQEFLS, 2017, pp. 33-42.

Ministère de l'Éducation, du Loisir et du Sport (MELS). La formation universitaire : les employeurs s'expriment, 2004,http://www.education.gouv.qc.ca/fileadmin/administration/librairies/documents/Ministere/acces_i nfo/Statistiques/Enquetes_Relance/Universite_Bac_Maitrise/Relance_universite_BacMai_Sondage_2004.pdf.

Ministère de l'Immigration et des Communautés culturelles (MICC). Échelle québécoise des niveaux de compétence en français des personnes immigrantes adultes, 2011, http://www.immigrationquebec.gouv.qc.ca/publications/fr/langue-francaise/Echelle-niveaux-competences.pdf.

Norris, John M. "Task-based teaching and testing." The handbook of language teaching, 2009, pp. 578-594. 
Michaud, Gabriel; Desaulniers, Annie et Juliane Bertrand. "L'Enseignement des langues par la tâche : le cas d'un cours visant les compétences informationnelles.» Nouvelle Revue Synergies Canada, №11 (2018)

Nunan, David. Designing Tasks for the Communicative Classroom. Cambridge University Press, 1989.

---. Learner-Centered English Language Education: The Selected Works of David Nunan. Routledge, 2012.

Richer, Jean-Jacques. La didactique des langues interrogée par les compétences. E.M.E. \& InterCommunications, 2012.

Samuda, Virginia et Martin Bygate. Tasks in Second Language Learning. Palgrave MacMillan, 2008.

Skehan, Peter. A Cognitive Approach to Language Learning. Oxford University Press, 1998.

Université du Québec. Normes de compétences et indicateurs de performance : contenus, stratégies et outils pédagogiques (un aperçu). 2004, http://ptc.uquebec.ca/pdci/system/files/documents/administration/ annexe_iii-pdci.pdf.

Université du Québec à Montréal (UQAM), Service des bibliothèques FLS - Compétences informationnelles Phase documentaire, 2016, http://guides.bibliotheques.uqam.ca/themes/159-FLS---Competencesinformationnelles---La-phase-documentaire?tab=970.

Van den Branden, Kris. Task-Based Language Education: From Theory to Practice. Cambridge University Press, 2006.

\section{Dictionnaires en ligne}

Le Petit Robert, 2016, http://pr.bvdep.com.proxy.bibliotheques.uqam.ca:2048/login_.asp

USITO, mise à jour du 9 février 2016, https://www-usitocom.proxy.bibliotheques.uqam.ca:2443/dictio/\#/babillard 